\title{
Synthesis and characterization of layered oriented hydrogen titanate micro-tube films
}

\author{
H ZHAO*, J Z PAN, S Y DU and C H CHEN \\ College of Environmental and Chemical Engineering, Dalian Jiaotong University, Dalian 116028, China
}

MS received 24 February 2009; revised 23 September 2009

\begin{abstract}
Continuous and uniform films of oriented hydrogen titanate tubes with diameters, c.a. $200 \mathrm{~nm}$, were prepared directly via porous anodic aluminum oxides (AAO) templates without any further removal of templates. Simple impregnation method was applied with aqueous solution titanium tetrafluoride $\left(\mathrm{TiF}_{4}\right)$ as the precursor to form the titania sol-gel. Scanning electron microscope (SEM) results show that the films are uniform, tubes highly oriented and even in length, diameter and morphology. Hydrogen trititanate $\left(\mathrm{H}_{2} \mathrm{Ti}_{3} \mathrm{O}_{7}\right)$ phase is proved by X-ray diffraction (XRD) patterns. Layered films of oriented tubes are fabricated when impregnation time is well controlled. The formation mechanism is drawn with the help of energy dispersion spectra (EDS). Due to the existence of $F^{-}$, the hydrolysis of $\mathrm{Ti}^{4+}$ is relatively slow along inner channel walls of AAO, which eventually forms compact tube arrays. The solubility among different parts of AAO template is of big difference in acidic surrounding. Layered films of oriented tubes are, therefore, fabricated when impregnation time is well controlled. The channel size of the AAO template takes control in deciding the pore size of the titania micro-tubes and reaction time, the length of the tubes.
\end{abstract}

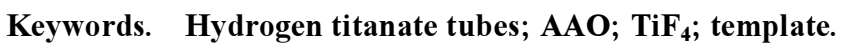

\section{Introduction}

Nano-structured materials have received much attention because of their novel properties. One-dimensional (1D) materials are an important category of nano-structured materials and have been widely studied yielding various special structures such as nano-tubes, nano-rods, nanowires and nano-belts. The titanium nano-oxides are of particular interest because of their high efficiencies in photocatalysis (Cardona et al 2004), solar cell (Graetzel and O'Regan 1991), photoelectrolysis (Mor et al 2005), dye sensitized solar cells (Zhu et al 2007), etc. The methods of preparing 1D-structured titania include mainly template synthesis (Hoyer 1996), supra-molecular assembles (Kobayashi 2000), hydrothermal synthesis (Hosono et al 2004) and inductive synthesis (Zhu and Ding 1999). However, thin films and coatings of oriented nanostructures are often more desirable for applications involving catalysis, filtration, sensing, photovoltaic cells and electrodes. Low cost preparation remains a challenge, and reports of such films are a few (Wu et al 2005). Tian et al (2003) reported large oriented arrays and continuous films of $\mathrm{TiO}_{2}$-based micro-tubes by templateless method. $\mathrm{Wu}$ et al synthesized large area films consisting of ordered titania rods via direct oxidation (Wu 2004; Wu

*Author for correspondence (zhaohong@djtu.edu.cn, hoz22002@yahoo.com) et al 2002, 2005; Huang et al 2006; Wu and Qi 2007). Liu et al (2002) reported a secondary growth from the site seeds of AAO membrane surface formed polycrystalline $\mathrm{TiO}_{2}$ nanotubes, with $20-40 \mathrm{~nm}$ in outer diameter and $4-5 \mathrm{~nm}$ in inner diameter. Nevertheless a substrate is indispensable and atleast $60^{\circ} \mathrm{C}$ surrounding is necessary.

In this paper we report a one step template method to directly prepare large scaled continuous layered films of oriented hydrogen titanate micro-tubes. The channel size of the AAO template takes control in deciding the pore size of the hydrogen titanate micro-tubes as prepared and reaction time, the length of the tubes.

\section{Experimental}

The precursor solution $\left(0.04 \mathrm{~mol} \mathrm{~L}^{-1}\right)$ was prepared by dissolving $\mathrm{TiF}_{4}(\mathrm{AR})$ in deionized water. The $\mathrm{pH}$ value of the solution was adjusted to $1 \cdot 6-2 \cdot 3$ with $\mathrm{HCl}$ and $\mathrm{NH}_{4} \mathrm{OH}$. Porous anodic aluminum oxide (AAO) membranes (Whatman Anodisc 25) were etched in $\mathrm{H}_{3} \mathrm{PO}_{4}(6$ $\mathrm{wt} \%$ ) for $15 \mathrm{~min}$ at $45^{\circ} \mathrm{C}$ before employing as templates. The templates with straight channels of ca. $300 \mathrm{~nm}$ in nomination were immersed in $\mathrm{TiF}_{4}$ solution and maintained at $60^{\circ} \mathrm{C}$ for $6-24 \mathrm{~h}$. After the reaction, the sample was washed with deionized water and dried at $60^{\circ} \mathrm{C}$ for 1 day.

The as-prepared samples were characterized by a scanning electron microscope (JSM-6360 LV) equipped with 
an X-ray microanalyser to detect their morphology and composition. JEM-2000 EX was used to characterize fine structure of the sample. The crystalline phase was identified by X-ray diffraction (XRD) using a Rigaku RAD-C system. Specified surface areas and pore-size distribution of the sample were measured by $\mathrm{N}_{2}$ adsorption using BET and $\mathrm{BJH}$ methods, respectively with a quantachrome Autosob 1 apparatus. The UV-vis absorbance spectra were performed on JASCO-550 Spectrophotometer in the range of $190-700 \mathrm{~nm}$. It is necessary to highlight that all the samples characterized in the study have not gone through any further removal procedures of AAO membranes.

\section{Results and discussion}

Figure 1 shows the scanning electron microscopy image (SEM) of a porous anodic aluminum oxide membrane $(d=2.5 \mathrm{~cm})$. The pores are evenly distributed with a distorted hexagonal pattern, reflecting the surface morphology of porous layer (PL). The inset of figure 1 is the image of its backside, which corresponds to the barrier layer (BL). The average channel size is c.a. $300 \mathrm{~nm}$ in diameter and $50 \mu \mathrm{m}$ in length. The AAO membranes were used as templates to guide the orientation of the deposition to form hydrogen titanate tubes in the pores with the hydrolysis of $\mathrm{TiF}_{4}$ solution.

The morphology and composition of the sample after $12 \mathrm{~h}$ of reaction with the solution are displayed in figure 2. Figure 2(a) shows the top view of the sample, in which the nano-particles and nano-holes are clearly demonstrated. The channel diameter of the sample ranges from $100-250 \mathrm{~nm}$. It is a relatively smooth, clean, ordered and continuous film mainly composed of titania. Figure 2(b) shows the image of its cross-section at low magnitude. The clear-cut border of two-layer in figure 2(b) is obvious. Figure 2(c) shows the right part of the two layers and figure 2(d) the left part in high magnitude. Both parts are constituted by individual oriented tubes with smooth walls and evenly distributed pore size. The tube has an inner diameter of about $150 \mathrm{~nm}$ and an outer diameter of about $250 \mathrm{~nm}$ both in figures 2(c) and (d), which goes well with the template morphology. The tubes on the left part are longer $(25 \mu \mathrm{m})$ and more straight than those on the right part $(10 \mu \mathrm{m})$ and congregate at their ends on the border.

Figures 3(a) and (b) are the cross section images of samples after reaction for $18 \mathrm{~h}$ and $24 \mathrm{~h}$, respectively. The morphology of the sample after $18 \mathrm{~h}$ reaction has a similar structure to that of the sample after $12 \mathrm{~h}$ reaction, but morphology of the sample after $24 \mathrm{~h}$ reaction varies a lot from the above two. The variation of the left part in length is small, while that of the right part changes from about $12 \mu \mathrm{m}(12 \mathrm{~h})$ to $9 \mu \mathrm{m}(18 \mathrm{~h})$ and disappears eventually $(24 \mathrm{~h})$. Generally the tubes on the left part are more densely distributed than those on the right. Referred to the original template structure of AAO, it was assumed that hydrogen titanate tubes on left part are formed in the $B$-layer and the right in the $L$-layer.

It is well known that an AAO membrane is composed of three parts: $P$-layer, $B$-layer and hydrated layer (H-layer) (Pu et al 2002) and the decreasing sequence of the solubility in acidic surrounding is $B$-layer, $H$-layer and $P$-layer (Brito 2001). The tubes formed in the $B$-layer collapsed together with the layer easily when the template was being dissolved into the surrounding and hydrogen titanate tubes on the left part (formed in the $P$-layer) remained longer. After $12 \mathrm{~h}$ reaction, a layered film of hydrogen titanate tube is formed, but after $24 \mathrm{~h}$ reaction,
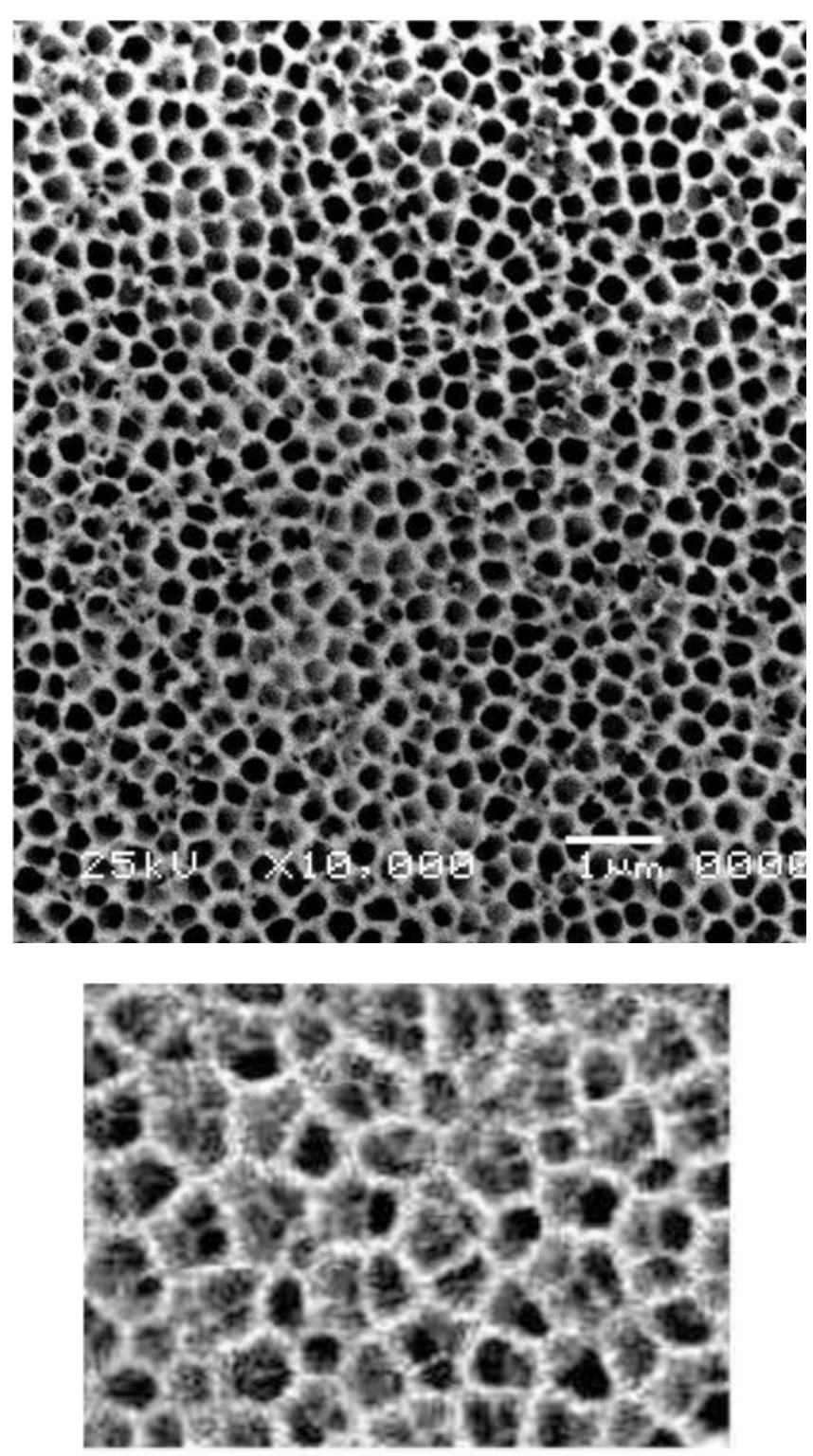

Figure 1. SEM image of porous type layer ( $P$ layer) of AAO template while the bottom image shows the barrier type layer (B layer). 

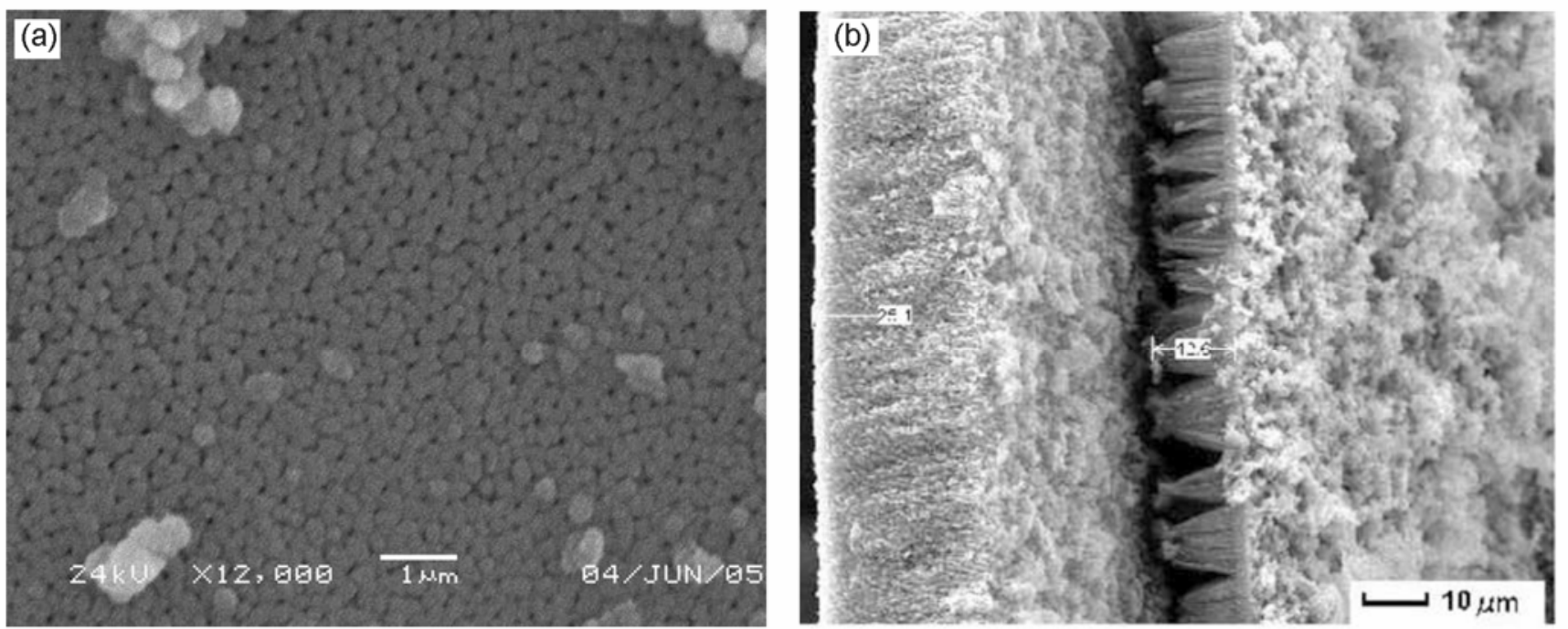

(b) inset (left)
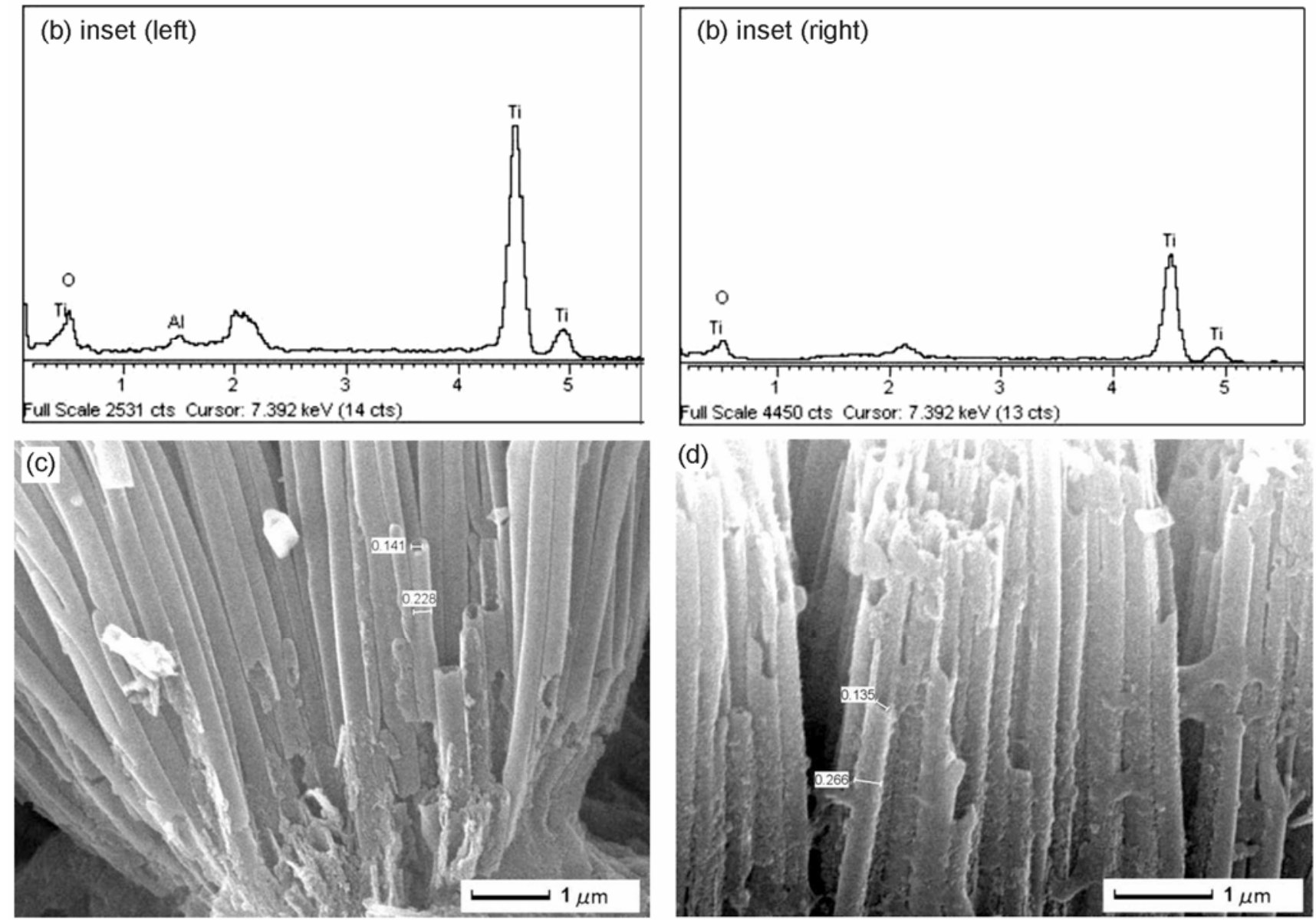

Figure 2. SEM images of hydrogen titanate maicro-tubes formed via AAO template after $12 \mathrm{~h}$ reaction. (a) The top view of the sample, (b) the cross section of the sample and EDS profiles corresponding to the right and the left part divided clearly by the border in between, (c) the enlargement of the right part and (d) the left part of $(\mathbf{b})$. The length $(\mu \mathrm{m})$ is labelled in white squares. The insets are EDS profiles corresponding to the parts those locate.

since hydrogen titanate tubes formed in the $B$-layer have dissolved totally, only a mono-layered film of hydrogen titanate tube formed in the $P$-layer, remained. It is obvi- ous that the layered films are achieved when impregnation time is well controlled. At the mean time, if the surrounding acidity is kept constant, the length of the 
tubes is also determined by the reaction time, which is a totally kinetic process.

The inset of figure 2(a) is its corresponding EDS profile, which shows clearly only $\mathrm{Ti}$ and $\mathrm{O}$ and confirms that the atomic ratio of these two elements is about $1: 2$ (The small peak at $2 \mathrm{kV}$ is caused by $\mathrm{Au}$ sputtering before
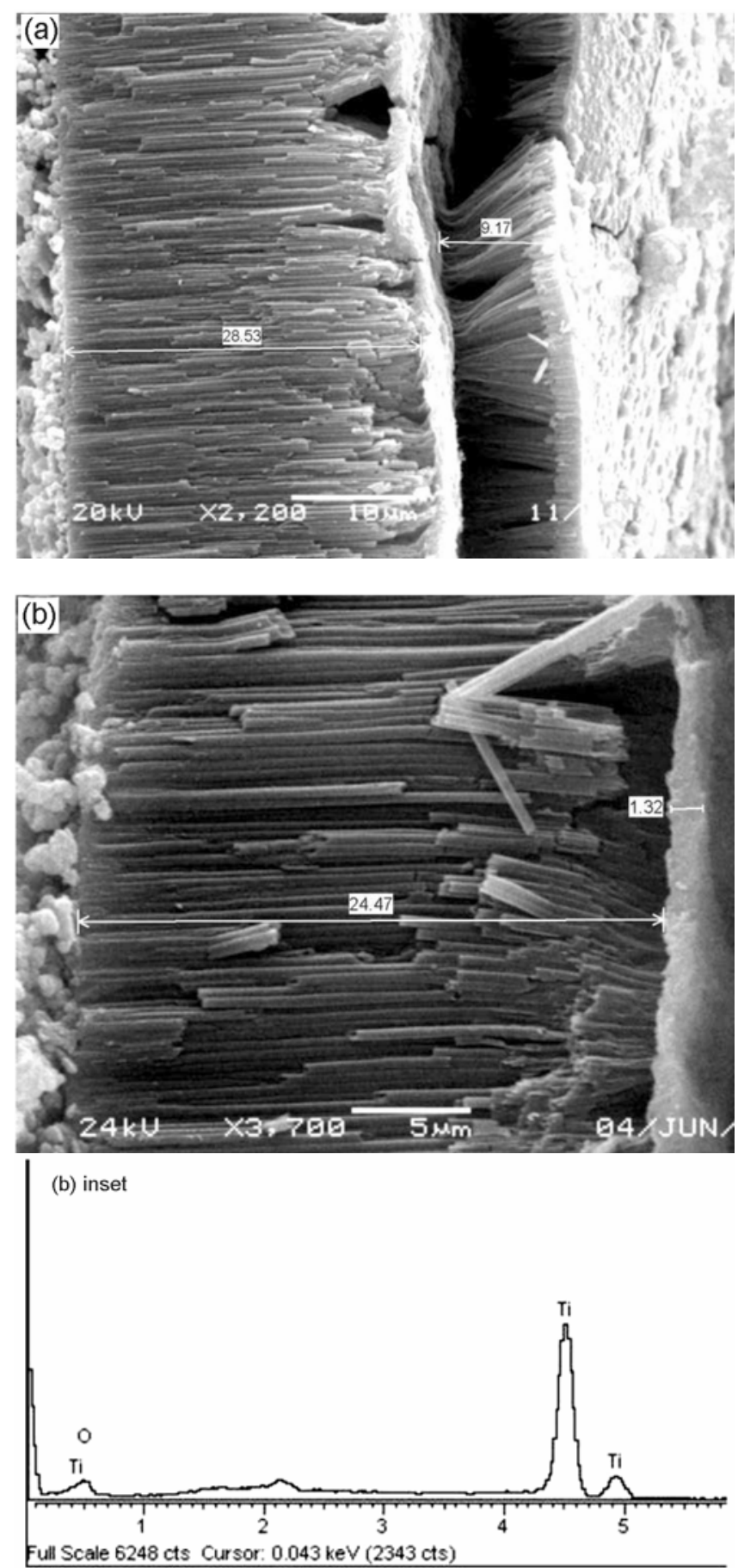

Figure 3. SEM images of cross-section of the sample after $18 \mathrm{~h}$ reaction (a) and $24 \mathrm{~h}$ reaction $(\mathbf{b})$. The inset of $(\mathbf{b})$ is the corresponding EDS profile. The length is indicated by arrows and nearby numbers $(\mu \mathrm{m})$. doing SEM for good conductivity). The insets of figures 2(c) and (d) show that the compositions of different layers of EDS are different. One layer (right) had Al element and the other (left) did not. The inset of figure 3(b) is its EDS analysis, which demonstrates that no $\mathrm{Al}$ and $\mathrm{F}$ elements are detected in both layers for the sample after $24 \mathrm{~h}$ reaction.

Since the channels of AAO template are completely through in both ends and much bigger in size, they can be thoroughly wetted by the $\mathrm{TiF}_{4}$ solution. It is generally acknowledged that the hydrolysis of $\mathrm{TiF}_{4}$ in solution occurs in a stepwise manner to produce crystalline titania, (1)

$$
\mathrm{TiF}_{4} \Rightarrow \mathrm{Ti}(\mathrm{OH})_{4-x} \mathrm{~F}_{x} \Rightarrow \mathrm{TiO}_{2} .
$$

According to theory of hard and soft acids and bases (HSAB), $\mathrm{TiF}_{4}$ is more stable than other inorganic slats of titanium, and therefore, the hydrolysis of $\mathrm{TiF}_{4}$ is relatively small and dense matured titania crystal easily forms. Similarly, the complex of $F^{-}$and $\mathrm{Al}^{3+}$ is stable, the hydrolysis must be accompanied by a dissolving process to form $\left(\mathrm{AlF}_{6}\right)^{3-}$ complex, which helps alumina walls dissolve from both sides in the acidic surrounding at low temperature. Hydrogen titanate tubes having formed in channels of $B$-layer collapsed together into the surrounding at the border of $H$-layer when the template was impregnated long enough $(24 \mathrm{~h})$, only continuous films of hydrogen titanate tubes on the left part (formed in the channels of $P$-layer) remained. Subsequently $\mathrm{TiF}_{4}$ is further hydrolyzed along the inner walls of the template channels, while the outer walls of the template channels are dissolved into the acidic surrounding.

Figure 4 shows the XRD pattern of hydrogen titanate tubes obtained from AAO template method. Even the amorphous AAO affects the peak shapes of titania, it still shows no signal of aluminum or aluminum oxide at all.

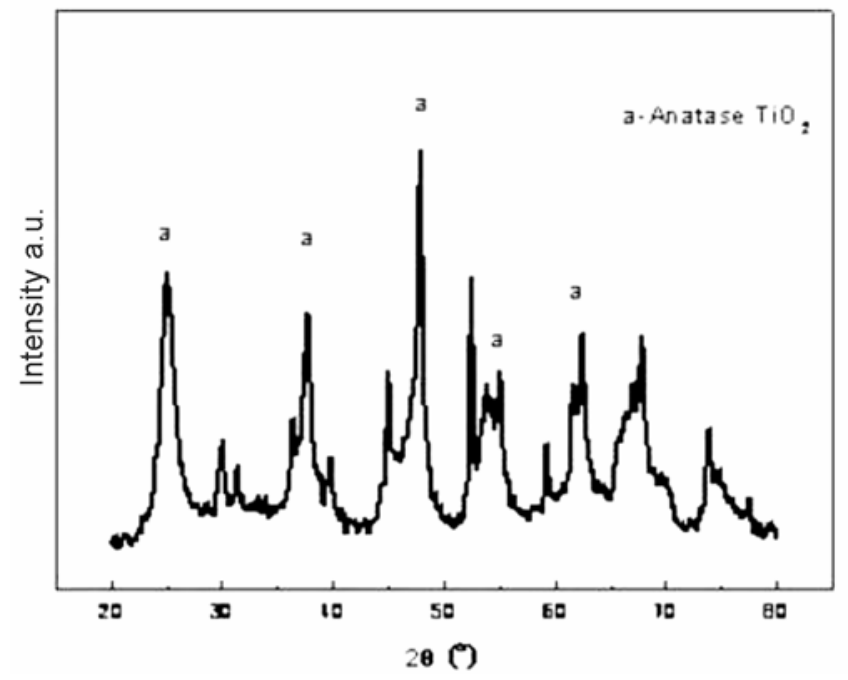

Figure 4. XRD pattern of the sample after $24 \mathrm{~h}$ reaction. 


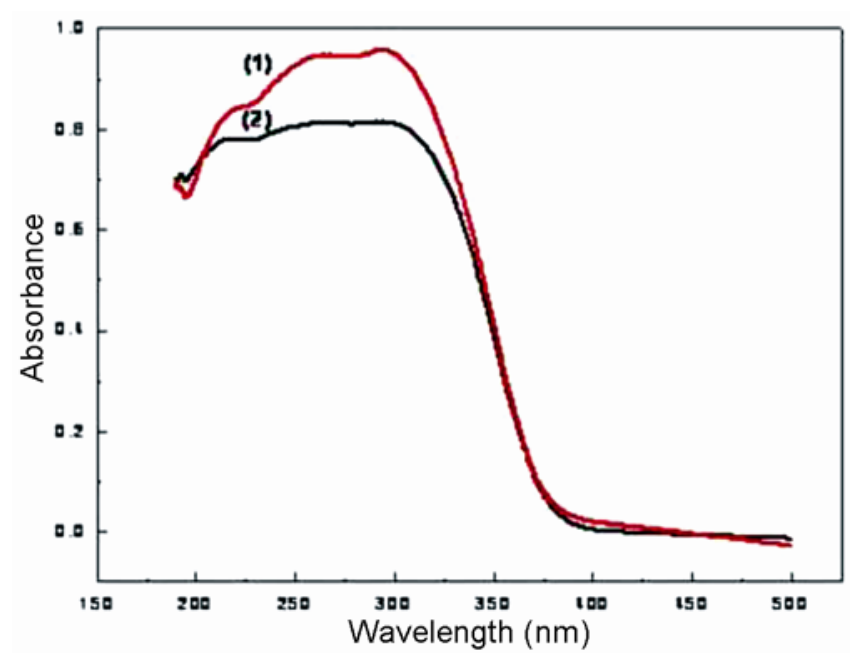

Figure 5. UV-Vis absorbance spectrum of $\mathrm{TiO}_{2}$ nanoparticles prepared by sol-gel method (c.a. $25 \mathrm{~nm}$ ) (1) and the sample after $24 \mathrm{~h}$ impregnation in the experiment (2).

By carefully comparing with PDF standards and analysing with JADE 5.0 software, hydrogen trititanate $\left(\mathrm{H}_{2} \mathrm{Ti}_{3} \mathrm{O}_{7}\right.$, PDF 47-0561) is identified along with some peaks of antase. The rutile structure as reported was not found in this experiment (Imai et al 1999).

The specific BET surface area of the sample is $68.5 \mathrm{~m}^{2} / \mathrm{g}$, which is quite smaller than the usual titania powder sample. Two reasons can be attributed to the small specific surface area. On the one hand, hydrogen titanate tubes are of big size in diameter (c.a. 200-300); on the other hand, the precursor solution goes through a slow hydrolysis procedure due to the competition for $F^{-}$ between $\mathrm{Ti}^{4+}$ and $\mathrm{Al}^{3+}$, heterogeneous nucleation occurs slowly on the alumina surface along the inner walls of the channels of AAO templates, which results into compact structure.

The photocatalytic activity of the deposited anatase was reported before (Hagfeldt and Graetzel 1995). UVvis spectra of the sample is different compared with titania nano-particles (c.a. $25 \mathrm{~nm}$ ) prepared by hydrolysis of $\mathrm{TiF}_{4}$ without any template (figure 5) in the wavelength region from $200-350 \mathrm{~nm}$. There are several weak peaks in the spectrum in this region of hydrogen titanate tubes, which implies different light absorption and propagation characteristics of the as prepared sample from $\mathrm{TiO}_{2}$ nanoparticles (c.a. $25 \mathrm{~nm}$ ). The appearance of $\mathrm{H}_{2} \mathrm{Ti}_{3} \mathrm{O}_{7}$ structure accounts for some difference. It is also possible the aligned porosity and oriented nature make the hydrogen titanate tube arrays more interesting. The hydrogen titanate tubes, hereforth, are expected to facilitate vectorial charge transfer between interfaces (Grimes 2007).

\section{Conclusions}

Without any further removal of the template, continuous and uniform film of hydrogen titanate tubes with diameters of c.a. $200 \mathrm{~nm}$ was prepared directly via porous anodic aluminum oxides (AAO) templates to guide the orientation. The channel size of AAO template takes control in deciding the pore size of the hydrogen titanate tubes as prepared and reaction time, the length of the tubes. The latter process is entirely kinetic when the surrounding acidity is kept constant. The structure with the aligned porosity, layered and oriented nature is expected to be useful as photo-catalysts and DSSC materials.

\section{Acknowledgement}

This work was financially supported by the Educational Department of Liaoning, China (B20040078).

\section{References}

Brito M E 2001 J. Mater. Sci. Lett. 202053

Cardona A I, Candal R, Sanchez B, Avila P and Rebollar M 2004 Energy 29845

Graetzel M and O'Regan B 1991 Nature 353737

Grimes C A 2007 J. Mater. Chem. 171451

Hagfeldt A and Graetzel M 1995 Chem. Rev. 9549

Hosono E, Fujihara S, Kakiuchi K and Imai H 2004 J. Am. Chem. Soc. 1267790

Hoyer P 1996 Langmuir 121411

Huang B, Wang M and Osaka A 2006 J. Am. Ceram. Soc. 89 2660

Imai H, Takei Y, Shimizu K, Matsuda M and Hirashima H 1999 J. Mater. Chem. 92971

Kobayashi S, Hanabusa K, Hamasaki N, Kimura M, Shirai H and Shinkai S 2000 Chem. Mater. 121523

Liu S M, Gan L M, Liu L H, Zhang W D and Zeng H C 2002 Chem. Mater. 141391

Mor G K, Shankar K, Paulose M, Varghese O K and Grimes C A 2005 Nano Lett. 5191

Pu L et al 2002 Chin. Phys. Lett. 19391

Tian Z R, Voigt J A, Liu J, Mackenzie B and Xu H 2003 J. Am. Ceram. Soc. 12512384

Wu J M 2004 J. Cryst. Growth 269347

Wu J M and Qi B 2007 J. Am. Ceram. Soc. 90657

Wu J M, Satoshi H, Tsurs K and Osaka A 2002 Cryst. Growth Des. 2147

Wu J M, Zhang T W, Zeng Y W, Satoshi H, Tsuru K and Osaka A 2005 Langmuir 216995

Zhu K, Neale N R, Miedaner A and Frank A J 2007 Nano Lett. 769

Zhu Y C and Ding C X 1999 Nanostruct. Mater. 11427 